The Journal of $\mathbf{N}_{\text {onlinear }} \mathbf{S}_{\text {cience and }}$ Applications

http://www.tjnsa.com

\title{
COMMON FIXED POINT THEOREMS FOR HYBRID MAPPINGS SATISFYING GENERALIZED CONTRACTIVE CONDITIONS
}

\author{
ABDELKRIM ALIOUCHE
}

\begin{abstract}
We prove common fixed point theorems in symmetric spaces for two pairs of hybrid mappings using the concept of $T$-weakly and $S$-weakly commuting mappings satisfying generalized contractive conditions which generalize theorems of Aamri and El Moutawakil [J. Math. Anal. Appl., 270 (2002), 181-188.], Aamri and El Moutawakil [Appl. Math. E-notes., 3 (2003), 156-162.] and Aliouche [J. Math. Anal. Appl., 322 (2006), 796-802.].
\end{abstract}

\section{INTRODUCTION AND PRELIMINARIES}

Let $(X, d)$ be a metric space. For $x \in X$ and $A \subset X, d(x, A)=\inf \{d(x, y), y \in A\}$. Let $C B(X)$ be the set of all nonempty closed and bounded subsets of $X$. Let $H$ be the Hausdorff metric with respect to $d$ defined by

$$
H(A, B)=\max \left\{\sup _{a \in A} d(a, B), \sup _{b \in B} d(A, b)\right\} \text { for all } A, B \in C B(X) .
$$

Let $f: X \rightarrow X$ be a single-valued mapping and $T: X \rightarrow C B(X)$ be a multi-valued mapping.

$f$ and $T$ are said to be commuting, see [9], in $X$ if for all $x \in X, f T x \subset T f x$.

$f$ and $T$ are said to be weakly commuting on $X$, see [17] and [18], if for all $x \in X, f T x \in C B(X)$ and

$$
H(f T x, T f x) \leq d(f x, T x)
$$

Date: Received: 23 October 2008; Accepted: 24 November 2008.

2000 Mathematics Subject Classification. Primary 47H10; Secondary 54H25.

Key words and phrases. Hybrid mappings, T-weakly commuting, property (E.A), common property (E.A), common fixed point, symmetric space. 
$f$ and $T$ are said to be compatible, see [10] and [13], if for all $x \in X, f T x \in$ $C B(X)$ and

$$
\lim _{n \rightarrow \infty} H\left(f T x_{n}, T f x_{n}\right)=0
$$

whenever $\left\{x_{n}\right\}$ is a sequence in $X$ such that $\lim _{n \rightarrow \infty} f x_{n}=t \in A=\lim _{n \rightarrow \infty} T x_{n}$ for some $t \in X$ and $A \in C B(X)$.

$f$ and $T$ are noncompatible if $f T x \in C B(X)$ for all $x \in X$ and there exists at least one sequence $\left\{x_{n}\right\}$ in $X$ such that $\lim _{n \rightarrow \infty} T x_{n}=A \in C B(X)$ and $\lim _{n \rightarrow \infty} f x_{n}=t \in A$, but $\lim _{n \rightarrow \infty} H\left(f T x_{n}, T f x_{n}\right)$ either non-zero or does not exist.

Commuting implies weakly commuting implies compatible, but the converse is not true in general, see [13.

$f$ and $T$ are said to be weakly compatible, see [11], if they commute at their coincidence points; i.e., $f x \in T x$ implies that $f T x=T f x$.

$f$ and $T$ are said to be $(I T)$-commuting at $x \in X$, see [20], if $f T x \subset T f x$.

$f$ and $T$ are said to be $R$-weakly commuting at $x \in X$, see [19], if $f T x \in$ $C B(X)$ and there exists an $R>0$ such that

$$
H(f T x, T f x) \leq R d(f x, T x) .
$$

$f$ and $T$ are said to be pointwise $R$-weakly commuting on $X$ if for all $x \in X$, $f T x \in C B(X)$ and (1.1) holds.

It was proved in [20] that a pointwise $R$-weakly commuting hybrid pair is not weakly compatible in general. However, pointwise $R$-weak commutativity at coincidence points is equivalent to $(I T)$ commutativity.

$f$ is $T$-weakly commuting at $x \in X$, see [12, if $f f x \in T f x$.

For a hybrid pair $(f, T),(I T)$ commuting at coincidence points implies that $f$ is $T$-weakly commuting, but the converse is not true, see [12].

If $T$ is a single-valued mapping, then $T$-weak commutativity at coincidence points is equivalent to weak commutativity.

Let $f, T: X \rightarrow X$. The pair $(f, T)$ satisfies the property (E.A), see [1], if there exists a sequence $\left\{x_{n}\right\}$ in $X$ such that $\lim _{n \rightarrow \infty} f x_{n}=\lim _{n \rightarrow \infty} T x_{n}=t$ for some $t \in X$.

Let $f: X \rightarrow X$ and $T: X \rightarrow C B(X)$. The pair $(f, T)$ satisfies property (E.A), see [12, if there exists a sequence $\left\{x_{n}\right\}$ is a sequence in $X$ such that $\lim _{n \rightarrow \infty} f x_{n}=t \in A=\lim _{n \rightarrow \infty} T x_{n}$ for some $t \in X$ and $A \in C B(X)$.

Let $f, g, S, T: X \rightarrow X$. The pairs $(f, S)$ and $(g, T)$ satisfy a common property (E.A), see [14], if there exist two sequences $\left\{x_{n}\right\},\left\{y_{n}\right\}$ and $t \in X$ such that

$$
\lim _{n \rightarrow \infty} S x_{n}=\lim _{n \rightarrow \infty} f x_{n}=\lim _{n \rightarrow \infty} g y_{n}=\lim _{n \rightarrow \infty} T y_{n}=t .
$$

If $S=T$ and $g=f$ in (1.2), we obtain the definition of property (E.A) for single-valued mappings.

Let $f, g: X \rightarrow X$ and $S, T: X \rightarrow C B(X)$. The pairs $(f, S)$ and $(g, T)$ satisfy a common property (E.A), see [14], if there exist two sequences $\left\{x_{n}\right\},\left\{y_{n}\right\}, t \in X$ and $A, B \in C B(X)$ such that

$$
\lim _{n \rightarrow \infty} S x_{n}=A, \lim _{n \rightarrow \infty} T y_{n}=B, \lim _{n \rightarrow \infty} f x_{n}=\lim _{n \rightarrow \infty} g y_{n}=t \in A \cap B .
$$


If $S=T$ and $g=f$ in (1.3), we obtain the definition of property (E.A) for multi-valued mappings.

It has been observed by Hicks and Rhoades [8] that some of the defining properties of the metric are not needed in the proofs of certain metric theorems. Motivated by this fact, they established some common fixed point Theorems in symmetric spaces and proved that very general probabilistic structures admit a compatible symmetric or semi-metric.

Recall that a symmetric on a set $X$ is a nonnegative real valued function $d$ on $X \times X$ such that

(i) $d(x, y)=0$ if and only if $x=y$,

(ii) $d(x, y)=d(y, x)$.

Let $d$ be a symmetric on a set $X$ and for $r>0$ and any $x \in X$, let $B(x, r)=$ $\{y \in X: d(x, y)<r\}$. A topology $t(d)$ on $X$ is given by $U \in t(d)$ if and only if for each $x \in U, B(x, r) \subset U$ for some $r>0$. A symmetric $d$ is a semi-metric if for each $x \in X$ and each $r>0, B(x, r)$ is a neighborhood of $x$ in the topology $t(d)$. Note that $\lim _{n \rightarrow \infty} d\left(x_{n}, x\right)=0$ if and only if $x_{n} \rightarrow x$ in the topology $t(d)$.

The following two axioms were given by Wilson [22]. Let $(X, d)$ be a symmetric space.

(W.3) Given $\left\{x_{n}\right\}, x$ and $y$ in $X$,

$\lim _{n \rightarrow \infty} d\left(x_{n}, x\right)=0$ and $\lim _{n \rightarrow \infty} d\left(x_{n}, y\right)=0$ imply $x=y$.

(W.4) Given $\left\{x_{n}\right\},\left\{y_{n}\right\}$ and $x$ in $X$,

$\lim _{n \rightarrow \infty} d\left(x_{n}, x\right)=0$ and $\lim _{n \rightarrow \infty} d\left(x_{n}, y_{n}\right)=0$ imply that $\lim _{n \rightarrow \infty} d\left(y_{n}, x\right)=0$

It is easy to see that for a semi-metric $d$, if $t(d)$ is Hausdorff, then (W.3) holds.

Now, we define the Hausdorff distance in a symmetric space.

Definition $1.1([7])$. Let $A$ be a nonempty subset of $X . A$ is said to be $d$-closed iff $A^{-d}=A$, where

$A^{-d}=\{x \in X: d(x, A)=0\}$ and $d(x, A)=\inf \{d(x, y): y \in A\}$.

$A$ is said to be $d$-bounded iff $\delta_{d}(A)<\infty$ where $\delta_{d}(A)=\sup \{d(x, y): x, y \in A\}$.

The following definition is a generalization of the well-known the Hausdorff distance to the setting of a symmetric case.

Definition $1.2([7])$. Let $C B(X)$ be the set of all nonempty $d$-closed and $d$-bounded subsets of $X$. Consider the function $H$ defined by

$$
H(A, B)=\max \left\{\sup _{a \in A} d(a, B), \sup _{b \in B} d(A, b)\right\} \text { for all } A, B \in C B(X) .
$$

It is easy to see that $(C B(X), H)$ is a symmetric space.

Example 1.3. Let $X=[1, \infty)$ and $d$ be a symmetric on $X$ defined by $d(x, y)=$ $e^{|x-y|}-1$ for all $x, y$ in $X$. Define $f: X \rightarrow X$ and $T: X \rightarrow C B(X)$ by $f(x)=2 x+1$ and $T x=[2,2 x+3]$. Consider the sequence $\left\{x_{n}\right\}$ such that $x_{n}=1+\frac{1}{n}, n=1,2, \ldots . t=3 \in[2,5]=A$. Clearly, $\lim _{n \rightarrow \infty} d\left(f x_{n}, 3\right)=0$ and $\lim _{n \rightarrow \infty} H\left(T x_{n}, A\right)=0$. Therefore, $(f, T)$ satisfies the property $(E . A)$.

Example 1.4. Let $X=\mathbb{R}$ with the above symmetric function $d$. It is easy to see that the condition (W.3) holds. Define $f, T: X-\rightarrow X$ by: $f x=x+1$ and 
$T x=\{x+2\}$, for all $x \in X$. Suppose that property (E.A) holds. Then there exists a sequence $\left\{x_{n}\right\}$ in $X$ satisfying $\lim _{n \rightarrow \infty} d\left(f x_{n}, t\right)=\lim _{n \rightarrow \infty} H\left(T x_{n}, A\right)=0$ for some $t \in X$. Therefore, $\lim _{n \rightarrow \infty} x_{n}=t-1$ and $A=\{t+2\}$ and obviously $t \notin A$. Hence $f$ and $T$ do not satisfy the property (E.A).

Example 1.5. Let $X=[1, \infty)$ and $d$ be a symmetric on $X$ defined by $d(x, y)=$ $e^{|x-y|}-1$ for all $x, y$ in $X$. Define $f, g: X \rightarrow X$ and $S, T: X \rightarrow C B(X)$ by $f x=2+\frac{x}{3}, g x=2+\frac{x}{2}, S x=[1,2+x]$ and $T x=\left[3,3+\frac{x}{2}\right]$. Consider the sequences $\left\{x_{n}\right\}$ and $\left\{y_{n}\right\}$ such that $x_{n}=3+\frac{1}{n}, y_{n}=2+\frac{1}{n}, n=1,2, \ldots$ Clearly, $t=3, A=[1,5], B=[3,4], t \in A \cap B$ and

$$
\lim _{n \rightarrow \infty} H\left(S x_{n}, A\right)=\lim _{n \rightarrow \infty} H\left(G y_{n}, B\right)=\lim _{n \rightarrow \infty} d\left(f x_{n}, 3\right)=\lim _{n \rightarrow \infty} d\left(g y_{n}, 3\right)=0 .
$$

Therefore, $(f, S)$ and $(g, T)$ satisfy a common property (E.A).

Definition 1.6. Let $(X, d)$ be a symmetric space. We say that $(X, d)$ satisfies the property (H.E) if given $\left\{x_{n}\right\},\left\{y_{n}\right\}$ and $x$ in $X$

$$
\lim _{n \rightarrow \infty} d\left(x_{n}, x\right)=0 \text { and } \lim _{n \rightarrow \infty} d\left(y_{n}, x\right)=0 \text { imply } \lim _{n \rightarrow \infty} d\left(x_{n}, y_{n}\right)=0 \text {. }
$$

Example 1.7. (i) Every metric space $(X, d)$ satisfies the property (H.E).

(ii) Let $X=[0,+\infty)$ with the symmetric function $d$ defined in Example 1.3.

It is easy to see that the symmetric space $(X, d)$ satisfies the property (H.E).

Several authors have proved fixed point theorems and common fixed point theorems for mappings satisfying contractive conditions of integral type, see [3], [5], 6], [16] and [21] .

Recently, Zhang [23] and Aliouche [4] proved common fixed point theorems using new generalized contractive conditions in metric spaces. These theorems extended well-known results in [5], [6], [16] and [21].

Let $A \in(0, \infty], R_{A}^{+}=[0, A)$. Let $F: R_{A}^{+} \rightarrow \mathbb{R}$ satisfying

(i) $F(0)=0$ and $F(t)>0$ for each $t \in(0, A)$,

(ii) $F$ is nondecreasing on $R_{A}^{+}$,

(iii) $F$ is continuous.

Define $\digamma[0, A)=\{F: F$ satisfies (i)-(iii) $\}$.

The following Lemma was proved in [23]

Lemma 1.8. Let $A \in(0,+\infty], F \in \digamma[0, A)$. If $\lim _{n \rightarrow \infty} F\left(\epsilon_{n}\right)=0$ for $\epsilon_{n} \in R_{A}^{+}$, then $\lim _{n \rightarrow \infty} \epsilon_{n}=0$.

The following examples were given in [23].

(i) Let $F(t)=t$, then $F \in \digamma[0, A)$ for each $A \in(0,+\infty]$.

(ii) Suppose that $\varphi$ is nonnegative, Lebesgue integrable on $[0, A)$ and satisfies

$$
\underset{0}{\lim } \varphi(t) d t>0 \text { for each } \epsilon \in(0, A) .
$$

Let $F(t)=\lim _{0}^{t} \varphi(s) d s$, then $F \in[0, A)$.

(iii) Suppose that $\psi$ is nonnegative, Lebesgue integrable on $[0, A)$ and satisfies

$$
\lim _{0}^{\epsilon} \psi(t) d t>0 \text { for each } \epsilon \in(0, A)
$$


and $\varphi$ is nonnegative, Lebesgue integrable on $\left[0, \lim _{0}^{A} \psi(s) d s\right)$ and satisfies

$$
\underset{0}{\lim } \varphi(t) d t>0 \text { for each } \epsilon \in\left(0, \lim _{0}^{A} \psi(s) d s\right) .
$$

Let $F(t)=\lim _{0}^{\lim _{0}^{t} \psi(s) d s} \varphi(u) d u$, then $F \in \digamma[0, A)$.

(iv) If $G \in[0, A)$ and $F \in \digamma[0, G(A-0))$, then a composition mapping $F \circ G \in$ $\digamma[0, A)$. For instance, let $H(t)=\lim _{0}^{F(t)} \varphi(s) d s$, then $H \in \digamma[0, A)$ whenever $F \in \digamma[0, A)$ and $\varphi$ is nonnegative, Lebesgue integrable on $\digamma[0, F(A-0))$ and satisfies

$$
\lim _{0}^{\epsilon} \varphi(t) d t>0 \text { for each } \epsilon \in(0, F(A-0)) .
$$

Let $A \in(0,+\infty], \psi: R_{A}^{+} \rightarrow \mathbb{R}_{+}$. Define $\Psi[0, A)=\{\psi: \psi(t)<t$ for each $t \in(0, A)\}$.

The following Theorems were proved in [1] and [3] respectively.

Theorem 1.9. Let $A, B, S$ and $T$ be self-mappings of a metric space $(X, d)$ such that

$$
d(A x, B y) \leq \phi(\max \{d(S x, T y), d(S x, B y), d(B y, T y)\})
$$

for all $x, y \in X$, where $0<\phi(t)<t$ for each $t>0$. Suppose that $A(X) \subset T(X)$ and $B(X) \subset S(X)$ and $(A, S)$ or $(B, T)$ satisfies the property (E.A). If the range of one of the mappings $A, B, S$ and $T$ is a complete subspace of $X$, then $A, B, S$ and $T$ have a unique common fixed point in $X$.

Theorem 1.10. Let $d$ be a symmetric for $X$ satisfying (W.3), (W.4) and (HE). Let $A, B, S$ and $T$ be self-mappings of $(X, d)$ such that

$$
\int_{0}^{d(A x, B y)} \varphi(t) d t \leq \phi\left(\int_{0}^{\max \{d(S x, T y), d(S x, B y), d(B y, T y)\}} \varphi(t) d t\right)
$$

for all $x, y \in X$, where $0<\phi(t)<t$ and $\varphi: \mathbb{R}_{+} \rightarrow \mathbb{R}_{+}$is a Lebesgue-integrable mapping which is summable, non-negative and such that

$$
\int_{0}^{\epsilon} \varphi(t) d t>0 \text { for all } \epsilon>0
$$

Suppose that $A(X) \subset T(X)$ and $B(X) \subset S(X),(A, S)$ and $(B, T)$ are weakly compatible and $(A, S)$ or $(B, T)$ satisfies the property (E.A). If the range of one of the mappings $A, B, S$ and $T$ is a complete subspace of $X$, then $A, B, S$ and $T$ have a unique common fixed point in $X$.

It is our purpose in this paper to extend Theorems 1.9 and 1.10 for two pairs of hybrid mappings; i.e., single-valued and multivalued mappings and prove common fixed point Theorems using generalized contractive conditions which generalize Theorems of [1], [2] and [3].

\section{MAIN RESUlTS}

Let $D=\sup \{d(x, y): x, y \in X\}$. Set $A=D$ if $D=\infty$ and $A>D$ if $D<\infty$. 
Theorem 2.1. Let $d$ be a symmetric for $X$ satisfying (W.3), (W.4) and (H.E). Let $f$ and $g$ be self-mappings of $X$ and $S, T$ be mappings from $X$ into $C B(X)$ such that

$$
\begin{gathered}
S(X) \subset g(X) \text { and } T(X) \subset f(X) \\
F(H(S x, T y)) \leq \psi(F(\max \{d(f x, g y), d(f x, T y), d(g y, T y)\}))
\end{gathered}
$$

for all $x, y \in X$, where $F \in F[0, A)$ and $\psi \in \Psi[0, F(A-0))$ for each $A \in(0,+\infty]$. Suppose that $(f, S)$ is $S$-weakly commuting at $u, f u=f$ fu for $u \in C(f, S):=$ set of coincidence points of $f$ and $S$ and $(g, T)$ is $T$-weakly commuting at $v, g v=g g v$ for $v \in C(g, T):=$ set of coincidence points of $g$ and $T$ and $(f, S)$ or $(g, T)$ satisfies the property (E.A). If one $f(X)$ or $g(X)$ is a closed subset of $X$, then $f, g, S$ and $T$ have a common fixed point in $X$.

Proof. Assume that the pair $(g, T)$ satisfies the property (E.A). Then, there exists a sequence $\left\{x_{n}\right\}$ in $X$ such that $\lim _{n \rightarrow \infty} d\left(g x_{n}, z\right)=0$ and $\lim _{n \rightarrow \infty} H\left(T x_{n}, A\right)=0$ for some $z \in X$ and $z \in A \in C B(X)$. By (H.E) we have $\lim _{n \rightarrow \infty} d\left(g x_{n}, T x_{n}\right)=0$. Since $T(X) \subset f(X)$, there exists a sequence $\left\{y_{n}\right\}$ in $X$ such that $f y_{n} \in T x_{n}$. Let us show that $\lim _{n \rightarrow \infty} H\left(S y_{n}, A\right)=0$. Suppose that $\lim \sup _{n \rightarrow \infty} H\left(S y_{n}, T x_{n}\right)>0$. Then, using (2.2) we have

$$
\begin{aligned}
\lim \sup _{n \rightarrow \infty} F\left(H\left(S y_{n}, T x_{n}\right)\right) \leq & \limsup _{\substack{n \rightarrow \infty\\
}} \psi\left(F\left(\max \left\{d\left(f y_{n}, g x_{n}\right), d\left(f x_{n}, T x_{n}\right)\right\}\right)\right) \\
= & \lim \sup _{n \rightarrow \infty} \psi\left(F\left(d\left(g x_{n}, T x_{n}\right)\right)\right) .
\end{aligned}
$$

Therefore

$$
\limsup _{n \rightarrow \infty} \psi\left(F\left(d\left(g x_{n}, T x_{n}\right)\right)\right)>0
$$

which is a contradiction. Then

$$
\lim \sup _{n \rightarrow \infty} F\left(H\left(S y_{n}, T x_{n}\right)\right)=0
$$

and Lemma 1.8 implies that $\lim _{n \rightarrow \infty} H\left(S y_{n}, T x_{n}\right)=0$. By (W.4), we deduce that $\lim _{n \rightarrow \infty} H\left(S y_{n}, A\right)=0$.

Suppose that $f(X)$ is a complete subspace of $X$. Then, $z=f u$ for some $u \in X$.

We claim that $f u \in S u$. Using (2.2) we get

$$
\begin{aligned}
F\left(H\left(S u, T x_{n}\right)\right) & \leq \psi\left(F\left(\max \left\{d\left(f u, g x_{n}\right), d\left(f u, T x_{n}\right), d\left(g x_{n}, T x_{n}\right)\right\}\right)\right) \\
& <F\left(\max \left\{d\left(f u, g x_{n}\right), d\left(f u, T x_{n}\right), d\left(g x_{n}, T x_{n}\right)\right\}\right) .
\end{aligned}
$$

Letting $n \rightarrow \infty$ we obtain

$$
\lim _{n \rightarrow \infty} F\left(H\left(S u, T x_{n}\right)\right)=0
$$

and Lemma 1.8 implies that $\lim _{n \rightarrow \infty} H\left(S u, T x_{n}\right)=0$. By (W.3) we have $f u \in S u=A$. 
Since $S(X) \subset g(X)$, there exists $v \in X$ such that $g v \in S u$. We claim that $g v \in T v$. If not, $(2.2)$ gives

$$
\begin{aligned}
F(d(g v, T v)) & \leq F(H(S u, T v)) \\
& \leq \psi(F(\max \{d(f u, g v), d(f u, T v), d(g v, T v)\})) \\
& <F(d(g v, T v))
\end{aligned}
$$

which is a contradiction. Hence, $g v \in T v$. Let us show that $z$ is a common fixed point of $f, g, S$ and $T$.

$S$-weak commutativity of $f$ and $S$ at $u$ implies that $f u=f f u \in S f u$; i.e., $z=f z \in S z$.

$T$-weak commutativity of $g$ and $T$ at $v$ implies that $g v=g g v \in T g v$; i.e., $z=g z \in T z$.

The proof is similar when $g(X)$ is assumed to be a closed subset of $X$ instead of $f(X)$.

Remark 2.2. If $S$ and $T$ are single-valued mappings in Theorem 2.1 and if we give to the function $F$ example $(i i)$ and others examples, we obtain Theorem 1.10 of [3] and several Corollaries.

If $S=T$ and $g=f$ in Theorem 3.1, we get the following Corollary.

Corollary 2.3. Let $d$ be a symmetric for $X$ satisfying (W.3) and (H.E). Let $f$ be a self-mapping of $X$ and $T$ be a mapping from $X$ into $C B(X)$ satisfying

$$
\begin{gathered}
T(X) \subset f(X) \\
F(H(T x, T y) \leq \psi(F(H(\max \{d(f x, f y), d(f x, T y), d(f y, T y)))
\end{gathered}
$$

for all $x, y \in X$, where $F \in \digamma[0, A)$ and $\psi \in \Psi[0, F(A-0))$ for each $A \in(0,+\infty]$. Suppose that $(f, T)$ is $T$-weakly commuting at $u, f u=f f u$ for $u \in C(f, T):=$ set of coincidence points of $f$ and $T$ and $(f, T)$ satisfies the property (E.A). If $f(X)$ is a closed subset of $X$, then $f$ and $T$ have a common fixed point in $X$.

Remark 2.4. If $F(t)=t$ and $T$ is a single-valued mappings in Corollary 2.3, we obtain Theorem 2.1 of [2].

Corollary 2.5. Let $(X, d)$ be a metric space, $f$ and $g$ be self-mappings of $X$ and $S, T$ be mappings from $X$ into $C B(X)$ satisfying (2.1) and (2.2). Suppose that $(f, S)$ is $S$-weakly commuting at $u, f u=f f u$ for $u \in C(f, S):=$ set of coincidence points of $f$ and $S$ and $(g, T)$ is T-weakly commuting at $v, g v=g g v$ for $v \in C(g, T):=$ set of coincidence points of $g$ and $T$ and $(f, S)$ or $(g, T)$ satisfies the property (E.A). If $f(X)$ or $g(X)$ is a closed subset of $X$, then $f, g, S$ and $T$ have a common fixed point in $X$.

Remark 2.6. If $S$ and $T$ are single-valued mappings and the function $F$ takes example $(i i)$ in Corollary 2.5, we obtain Corollary 3 of [3].

If we give to the function $F$ examples $(i),(i i), i(i i)$ and $(i v)$ in Corollary 2.5. we obtain several Corollaries.

If $S$ and $T$ are single-valued mappings and $F(t)=t$ in Corollary 2.5, we obtain Theorem 1.9 of [1]. 
Theorem 2.7. Let d be a symmetric for $X$ satisfying (W.3), (W.4) and (H.E). Let $f$ and $g$ be self-mappings of $X$ and $S, T$ be mappings from $X$ into $C B(X)$ satisfying (2.2). Suppose that $(f, S)$ is $S$-weakly commuting at $u$, $f u=f f u$ for $u \in C(f, S):=$ set of coincidence points of $f$ and $S$ and $(g, T)$ is $T$-weakly commuting at $v, g v=g g v$ for $v \in C(g, T):=$ set of coincidence points of $g$ and $T$ and $(f, S)$ and $(g, T)$ satisfy a common property (E.A). If $f(X)$ and $g(X)$ are closed subsets of $X$, then $f, g, S$ and $T$ have a common fixed point in $X$.

Proof. Suppose that $(f, S)$ and $(g, T)$ satisfy a common property (E.A). Then, there exists two sequences $\left\{x_{n}\right\},\left\{y_{n}\right\}, z \in X$ and $A, B \in C B(X)$ such that

$\lim _{n \rightarrow \infty} H\left(S x_{n}, A\right)=\lim _{n \rightarrow \infty} H\left(T y_{n}, B\right)=\lim _{n \rightarrow \infty} d\left(f x_{n}, z\right)=\lim _{n \rightarrow \infty} d\left(g y_{n}, z\right)=0, z \in A \cap B$.

Assume that $f(X)$ and $g(X)$ are closed subsets of $X$. Then, $z=f u=g v$ for some $u, v \in X$.

We claim that $f u \in S u$. Using (2.2) we get

$$
\begin{aligned}
F\left(H\left(S u, T y_{n}\right)\right) & \leq \psi\left(F\left(\max \left\{d\left(f u, g y_{n}\right), d\left(f u, T y_{n}\right), d\left(g y_{n}, T y_{n}\right)\right\}\right)\right) \\
& <F\left(\max \left\{d\left(f u, g y_{n}\right), d\left(f u, T y_{n}\right), d\left(g y_{n}, T y_{n}\right)\right\}\right) .
\end{aligned}
$$

Letting $n \rightarrow \infty$ we obtain

$$
\lim _{n \rightarrow \infty} F\left(H\left(S u, T y_{n}\right)\right)=0
$$

and Lemma 1.8 implies that $\lim _{n \rightarrow \infty} H\left(S u, T y_{n}\right)=0$. By (W.3) we have $f u \in S u=B$.

The rest of the proof follows as in Theorem 2.1.

Corollary 2.8. Let $(X, d)$ be a metric space, $f$ and $g$ be self-mappings of $X$ and $S, T$ be mappings from $X$ into $C B(X)$ satisfying (2.2). Suppose that $(f, S)$ is $S$-weakly commuting at $u, f u=f f u$ for $u \in C(f, S):=$ set of coincidence points of $f$ and $S$ and $(g, T)$ is $T$-weakly commuting at $v$, gv $=$ ggv for $v \in$ $C(g, T):=$ set of coincidence points of $g$ and $T$ and $(f, S)$ and $(g, T)$ satisfy a common property (E.A). If $f(X)$ and $g(X)$ are closed subsets of $X$, then $f, g, S$ and $T$ have a common fixed point in $X$.

Remark 2.9. If we give to the function $F$ examples $(i),(i i),(i i i)$ and $(i v)$ in Theorem 2.7, we get several Corollaries.

If we give to the function $F$ examples $(i),(i i), i(i i)$ and $(i v)$ in Corollary 2.8 . we get several Corollaries.

Example 2.10. Let $X=[1, \infty)$ and $d$ be a symmetric on $X$ defined by $d(x, y)=$ $(x-y)^{2}$ for all $x, y$ in $X$. Define $f, g: X \rightarrow X$ and $S, T: X \rightarrow C B(X)$ by

$$
f(x)=x^{2}, g(x)=x^{4}, S x=[1, x+1] \text { and } T x=\left[1, x^{2}+1\right] .
$$

We have for all $x, y \in X$

$$
\begin{aligned}
d(f x, g y) & =\left(x^{2}-y^{4}\right)^{2} \\
& =\left(x+y^{2}\right)^{2}\left(x-y^{2}\right)^{2} \\
& \geq 4\left(x-y^{2}\right)^{2} \\
& =4 H(S x, T y)
\end{aligned}
$$


and so

$$
\begin{aligned}
H(S x, T y) & \leq \frac{1}{4} d(f x, g y) \\
& =\psi(d(f x, g y))
\end{aligned}
$$

Clearly, $A=D=\infty, F(t)=t, F \in \digamma[0, \infty), \psi(t)=\frac{t}{4}, \psi \in \Psi[0, \infty)$, $S(X)=g(X)=T(X)=f(X)=[1, \infty[,(f, S)$ is $S$-weakly commuting at 1 , $f 1=f^{2} 1=1,(g, T)$ is $T$-weakly commuting at $1, g 1=g^{2} 1=1$. Taking

$x_{n}=1+\frac{1}{n}$, the pair $(f, S)$ satisfies the property (E.A) with $t=1, A=[1,2]$ and $f(X)$ is a closed subset of $X$. Moreover, the pairs $(f, S)$ and $(g, T)$ satisfy a common property (E.A) with $t=1, A=B=[1,2]$ and $g(X)$ is a closed subset of $X$. Consequently, by Theorems 2.1 and 2.7, 1 is a common fixed point of $f, g, S$ and $T$.

\section{REFERENCES}

[1] M. Aamri and D. El Moutawakil, Some new common fixed point theorems under strict contractive conditions, J. Math. Anal. Appl., 270 (2002), 181-188. 1, 1, 1. 2.6

[2] M. Aamri and D. El Moutawakil, Common fixed points under contractive conditions in symmetric spaces, Appl. Math. E-notes., 3 (2003), 156-162. 1. 2.4

[3] A. Aliouche, A common fixed point Theorem for weakly compatible mappings in symmetric spaces satisfying a contractive condition of integral type, J. Math. Anal. Appl., 322 (2006), 796-802. 1, 1, 1, 2.2, 2.6

[4] A. Aliouche, Common fixed point theorems of Gregus type for weakly compatible mappings satisfying generalized contractive conditions, J. Math Anal. Appl., 341 (2008), 707-719. 1

[5] A. Branciari, A fixed point theorem for mappings satisfying a general contractive condition of integral type, Int. J. Math. Math. Sci., 29 (2002) 531-536. 1

[6] A. Djoudi and A. Aliouche, Common fixed point theorems of Gregus type for weakly compatible mappings satisfying contractive conditions of integral type, J. Math Anal. Appl., 329 (2007), 31-45. 1

[7] D. El Moutawakil, A fixed point theorem for multivalued maps in symmetric spaces, Applied Mathematics E-notes., 4 (2004), 26-32. 1.1. 1.2

[8] T. L. Hicks and B. E. Rhoades, Fixed point theory in symmetric spaces with applications to probabilistic spaces, Nonlinear Analysis., 36 (1999), 331-344. 1

[9] S. Itoh and W. Takahashi, Single-valued mappings, Multivalued mappings and fixed point theorems, J. Math. Anal. Appl., 59 (1977), 514-521. 1

[10] G. Jungck, Compatible mappings and common fixed points, Int. J. Math. Math. Sci., 9 (1986), 771-779. 1

[11] G. Jungck, Common fixed points for non-continuous non-self maps on non metric spaces, Far East J. Math. Sci., 4 (2) (1996), 199-215. 1

[12] T. Kamran, Coincidence and fixed points for hybrid strict contractions, J. Math. Anal. Appl., 299 (2004), 253-2-41. 1

[13] H. Kaneko and S. Sessa, Fixed point theorems for compatible multivalued and single valued mappings, Internat. J. Math. Math. Sci., 12 (1989), 257-262. 1

[14] W. Liu, J. Wu and Z. Li, Common fixed points of single-valued and multi-valued maps, Internat. J. Math. Math. Sci., 19 (2005), 3045-3055. 1. 1 
[15] R. P. Pant, Common fixed points of noncommuting mappings, J. Math. Anal. Appl., 188 (1994), 436-440.

[16] B. E. Rhoades, Two fixed-point theorems for mappings satisfying a general contractive condition of integral type, Int. J. Math. Math. Sci., 63 (2003), 4007-4013. 1

[17] S. Sessa, On a weak commutativity condition of mappings in fixed point considerations, Publ. Inst. Math., (Beograd), 32 (1982), 149-153. 1

[18] S. Sessa, M. S. Khan and M. Imdad, A common fixed point theorem with a weak commutativity condition, Glas. Math. Ser. III 21 (1986), 225-235. 1

[19] N. Shahzad and T. Kamran, Coincidence points and $R$-weakly commuting maps, Arch. Math. (Brno) 37 (2001), 179-183. 1

[20] S. L. Singh and S. N. Mishra, Coincidence and fixed points of non-self hybrid contractions, J. Math. Anal. Appl., 256 (2001), 486-497. 11, 1

[21] P. Vijayaraju, B. E. Rhoades, R. Mohanraj, A fixed point theorem for a pair of maps satisfying a general contractive condition of integral type, Int. J. Math. Math. Sci., 15 (2005), 2359-2364. 1

[22] W. A. Wilson, On semi-metric spaces, Amer. J. Math., 53 (1931), 361-373. 1

[23] X. Zhang, common fixed point theorems for some new generalized contractive type mappings, J. Math. Anal. Appl., 333 (2007), 780-786. 1 , 1

Department of Mathematics, University of Larbi Ben M'Hidi, Oum-El-Bouaghi, 04000, Algeria.

E-mail address: alioumath@gmail.com 